\title{
Symmetries of the Doi kinetic theory for nematic polymers of arbitrary aspect ratio: at rest and in linear flows
}

\author{
M. Gregory Forest \& Ruhai Zhou \\ Department of Mathematics \\ University of North Carolina at Chapel Hill \\ Chapel Hill, NC 27599-3250
}

\author{
Qi Wang \\ Department of Mathematics \\ Florida State University \\ Tallahassee, FL 32306
}

February 6, 2002

\begin{abstract}
The Doi theory [5] has successfully modeled the monodomain shear flow problem for rigid, rod-like nematic polymers. Numerical simulations of the Smoluchowski equation for the orientational probability distribution function (pdf) $([15,16,20,21,17,7,11])$ have predicted: monodomain attractors in regions of the 2-parameter space of nematic concentration $N$ and shear rate $\dot{\gamma}$; and bifurcation curves of monodomain transitions. Theoretical work $[29,13,14,30,23,28,19,20,21,22]$ has focused on approximate constructions of pdf solutions in various linear flow regimes. An alternative approach undertaken here is to develop symmetries of the Smoluchowski equation which imply global properties that all solutions must obey, and potentially explain important numerical or experimental phenomena. In [8] the authors developed flow-nematic symmetries for mesoscopic tensor approximations of the extended Doi theory for arbitrary molecular aspect ratio [32]. In this paper we generalize these symmetries to the Smoluchowski equation in explicit constructive form: the orientational degeneracy of quiescent nematic liquids is a continuous $O(3)$ symmetry, which is spontaneously broken by flow; in simple shear, a discrete reflection symmetry survives; a rod-discotic symmetry is established between monodisperse nematic liquids of reciprocal aspect ratio; and finally, a (flow, aspect ratio) symmetry relates the linear-flow response of finite aspect-ratio liquids to a related linear flow of an infinite aspect-ratio rod or discotic liquid. These symmetries are evident in solution diagrams from recent kinetic [7, 11, 9] and mesoscopic simulations [8] of the shear flow problem, and further explain deeper symmetries of the stable and unstable manifolds of numerical solution branches.
\end{abstract}




\section{Introduction}

Numerical simulations, largely based on spherical harmonic expansions $[23,15,24,16$, $22,17,7,11]$, have been instrumental in establishing agreement between experimental phenomena of nematic polymers and the Doi kinetic theory $[5,6]$ for flowing polymeric liquid crystals. Most notably, monodomain attracting states and their transitions versus shear rate have been correlated with rheological features of sign changes in normal stress differences and structural changes in apparent viscosity and shear stress. These correlations with experiment rely first and foremost on accurate simulation of the Smoluchowski equation for the molecular orientational probability distribution function, and then on the identification of those solutions with monodomain steady or transient states. (The latter step involves projection of the orientational distribution function onto the second-moment of the distribution.)

We note for example that the so-called logrolling and kayaking solutions, whose major director is respectively aligned with or oscillates around the vorticity axis in simple shear, were first exhibited with kinetic theory simulations in the early 90's [15, 16]. Attracting states which align or oscillate strictly between the vorticity axis and shearing plane have only recently been discovered $[7,11]$. The final step in relating theory to experiment, once the phase diagram of attracting states is established, is to evaluate constitutive laws for rheological properties along each stable solution branch. The brunt of the difficulty therefore lies in the accurate computation of the full flow-phase diagram of all individual monodomain attractors, their classification as steady or unsteady, stable or unstable, the multiplicity (continuous or discrete) of solution branches, and their transitions (i.e., bifurcations) which may occur by varying shear rate, or strength of the nematic potential (usually modeled as a concentration parameter), or other recent model parameters included in the extended Doi kinetic theory such as molecular aspect ratio (cf., [32]).

Renewed interest in monodomain dynamics of rigid macromolecular fluids is compelled by recent detailed kinetic theory simulations of Faraoni et al. [7] and Grosso et al. [11] for the three-dimensional orientational distribution function. These studies reveal striking new kinetic theory phenomena, as well as clarify previously reported results, in the monodomain response of rigid thin rods to simple shear:

- a discrete number of stable and unstable solution branches for each fixed shear rate;

- various bifurcations among these branches, including a period-doubling route to chaotic dynamics in a window of intermediate shear rates for a narrow range of nematic concentration; and

- a reflection-symmetry of out-of-plane states (both steady and periodic) which lead, 
among other properties, to parameter regimes of bi-stable orientational response.

Coincident with these Doi kinetic theory results, bifurcation diagrams of the authors [8] for a host of Doi mesoscopic second-moment tensor models for finite-aspect-ratio nematic fluids [32] reproduce each of these prominent features; details of the first two properties vary with closure rule and the molecular aspect ratio, while the latter property is common among all closures. Theoretical studies based on symmetry considerations of Landau-deGennes tensor models [4] by Chillingworth, Sluckin, Vicente Alonso, and Wheeler [3, 31] provide explanations of surprising shear-induced phenomena such as families of steady out-of-plane states seen numerically in kinetic [7,9] and mesoscopic [8] simulations.

From the theory of dynamical systems one knows that global features such as highlighted above, i.e., properties of the entire phase space of solutions, are often associated with symmetries. In [8] we developed various symmetries which were shared by all eight mesoscopic tensor models, i.e., properties which were apparently robust to closure approximation. We applied these mesoscopic symmetries to rigorously explain two features shared by all mesoscopic models: a discrete number of monodomains are selected in the weak shear limit, and the reflection-symmetry of the entire orientation tensor phase space. The evidence was overwhelming that these symmetries were kinetic theory properties, which we now prove in the present article. We remark that the proof of these results for the full Smoluchowski integral-differential equation follows that of the mesoscopic tensor ordinary differential equation, yet it is unlikely that we would have recognized these symmetries, or their proof if we did recognize them, without the relative simplicity of the five-dimensional, second-moment tensor, approximate models. The same remark applies to the equivariant bifurcation theory approach [3, 31], which has to be developed first in finite dimensions before extension to the infinite-dimensional Smoluchowski equation.

In addition to symmetries of the extended Doi kinetic theory for fixed aspect-ratio nematic liquids at rest and in simple shear, we also extend the mesoscopic symmetry properties in [8] that relate the orientational response of different aspect-ratio fluids. These results imply a one-to-one correspondence between the orientational response of all finite-aspectratio, monodisperse nematic liquids and a single, nearly infinite-aspect-ratio nematic liquid. Thus one can trade off the finite-aspect-ratio of the fluid by parametrically varying the straining component of the flow field with an extremely large aspect ratio fluid! Since we now establish this result for kinetic theory, it strongly compels experimental validation which we are pursuing with colleagues P. Mather, University of Connecticut, and K. Koelling, Ohio State University.

It must be underscored, however, that this one-to-one correspondence is for the orientational distribution functions of the respective fluids and flows, yet the corresponding rhe- 
ological responses are different! The disparate shear stress, apparent viscosity, and normal stress differences for identical monodomain flow-phase diagrams arise because of the strong dependence of Miesowicz viscosities on molecular aspect ratio (cf., [32]). Consequences of this and all symmetries developed below are deferred to subsequent communications (e.g., $[9])$.

\section{The extended Doi kinetic theory}

We first recall the kinetic theory for homogeneous nematic liquids relevant for our purposes [32]. Let $f(\mathbf{m}, t)$ be the probability distribution function (pdf) corresponding to the probability that the axis of revolution of the molecule is parallel to direction $\mathbf{m}(\|\mathbf{m}\|=1)$ at time $t$, where the lcp molecule is modeled as an axisymmetric ellipsoid of aspect ratio $r$ (length of the molecule symmetry axis divided by the radius of the transverse circular cross-section). The fluid velocity is denoted v. The Smoluchowski (kinetic) equation for $f(\mathbf{m}, t)$ is given by (e.g., $[6,2,32])$ :

$$
\frac{\partial f}{\partial t}=D_{r}(a) \mathcal{R} \cdot\left[\left(\mathcal{R} f+\frac{1}{k T} f \mathcal{R} V_{M S}\right)\right]-\mathcal{R} \cdot[\mathbf{m} \times \dot{\mathbf{m}} f]
$$

where $\frac{\partial}{\partial \mathbf{x}}=\nabla$ and $\mathcal{R}=\mathbf{m} \times \frac{\partial}{\partial \mathbf{m}}$ are the spatial and the rotational gradient operator, respectively;

$$
\dot{\mathbf{m}}=\mathbf{\Omega} \cdot \mathbf{m}+a[\mathbf{D} \cdot \mathbf{m}-\mathbf{D}: \mathbf{m m m}]
$$

is the Jeffery orbit of axisymmetric ellipsoidal molecules [12], $\mathbf{D}$ and $\boldsymbol{\Omega}$ are the rate of strain and vorticity tensors, respectively defined by

$$
\mathbf{D}=\frac{1}{2}\left(\nabla \mathbf{v}+\nabla \mathbf{v}^{T}\right), \Omega=\frac{1}{2}\left(\nabla \mathbf{v}-\nabla \mathbf{v}^{T}\right),
$$

$-1 \leq a \leq 1$ is the molecular shape parameter related to the molecule aspect ratio $r$ by

$$
a=\frac{r^{2}-1}{r^{2}+1}
$$

The coefficient $D_{r}$ is an averaged rotary diffusivity, taken to be constant to make contact with $[7,11]$; an orientation-dependent rotary diffusivity will not change the symmetry properties developed below, but will affect the phase diagram in the simple flows discussed herein; $k$ is the Boltzmann constant, $T$ is absolute temperature, and $V_{M S}$ is the Maier-Saupe intermolecular potential with strength proportional to the dimensionless polymer concentration $N$ :

$$
V_{M S}=-\frac{3 N k T}{2}\langle\mathbf{m m}\rangle: \mathbf{m m} .
$$


Here

$$
\langle(\bullet)\rangle=\int_{\|\mathbf{m}\|=1}(\bullet) f(\mathbf{m}, t) d \mathbf{m} .
$$

The average, or mesoscopic, molecular orientation is traditionally defined in terms of the second-moment of $f, \mathbf{M}$, or its traceless equivalent $\mathbf{Q}$, the mesoscopic orientation tensor:

$$
\mathbf{M}=\langle\mathbf{m m}\rangle, \quad \mathbf{Q}=\mathbf{M}-\frac{\mathbf{I}}{3} .
$$

The expression for the stress tensor [32] is not needed for the purposes of this paper, so we omit it.

These equations, coupled with momentum, mass and energy balance equations, constitute the extended Doi theory for finite-aspect-ratio nematic fluids. For isothermal, linear flow fields, these conservation laws are satisfied identically, and the full system "simplifies" to the homogeneous kinetic equation (1).

\section{Orientational degeneracy in hydrodynamic equilib- rium}

Without flow, the kinetic equation (1) reduces to

$$
\frac{\partial f}{\partial t}=D_{r}(a) \mathcal{R} \cdot\left[\left(\mathcal{R} f+\frac{1}{k T} f \mathcal{R} V_{M S}\right)\right]
$$

Let $\mathbf{U} \in O(3)$ denote any orthogonal transformation acting on the configuration space (the sphere $S^{2}$ ) of $\mathbf{m}$ (see [26]). We now state the basic fact:

Orientational degeneracy of quiescent nematic liquids. Every anisotropic solution of (8) generates a continuous family of solutions parametrized by the group $O(3)$ of orthogonal transformations. The isotropic equilibrium is isolated, i.e., a fixed point of the symmetry.

We give a proof next, then give an explicit algorithm to generate the group of solutions from any given fixed solution. We emphasize that this statement is not restricted to equilibrium distributions; it applies to every orbit through arbitrary initial orientational distributions. Thus the entire solution space is degenerate except for the lone isotropic state.

First, rewrite (8) into the form

$$
\frac{\partial f}{\partial t}=D_{r}(a) \mathcal{R} \cdot\left[\left(\mathcal{R} f+\frac{1}{k T} f \mathcal{R} V_{M S}\right)\right]=D_{r}(a) \frac{\partial}{\partial \mathbf{m}} \cdot\left[\frac{\partial}{\partial \mathbf{m}} f+\frac{1}{k T} f \frac{\partial}{\partial \mathbf{m}} V_{M S}\right] .
$$

In this proof, we consider a general class of intermolecular potentials $V$ all of which depend nonlocally on the pdf $f$ :

$$
V(\mathbf{m}, f(\cdot, t))=\int_{\mid \mathbf{m}^{\prime} \|=1} h\left(\mathbf{m}, \mathbf{m}^{\prime}\right) f\left(\mathbf{m}^{\prime}, t\right) d \mathbf{m}^{\prime},
$$


where $h\left(\mathbf{m}, \mathbf{m}^{\prime}\right)$ is a geometric expression for the excluded volume which must (on physical grounds) satisfy a basic duality relation:

$$
h\left(\mathbf{m}, \mathbf{U}^{T} \cdot \mathbf{m}^{\prime}\right)=h\left(\mathbf{U} \cdot \mathbf{m}, \mathbf{m}^{\prime}\right) .
$$

This class of potentials, and the symmetry property, includes Maier-Saupe and Onsager potentials as special cases:

$$
\begin{aligned}
& h_{M S}=-\frac{3}{2} N k T\left(\mathbf{m} \cdot \mathbf{m}^{\prime}\right)^{2}, \\
& h_{O}=\beta N k T\left\|\mathbf{m} \times \mathbf{m}^{\prime}\right\|,
\end{aligned}
$$

where $N$ is a strength parameter associated with a dimensionless molecular concentration, and $\beta$ is a parameter proportional to the volume of the molecule. The symmetry (11) states that the excluded-volume potential cannot tell the difference if one rotates $\mathbf{m}$ relative to $\mathbf{m}^{\prime}$ or vice versa. Here one can see that this property applies to any aspect-ratio molecular fluid for which a distinguished molecule direction is specified. For more complicated molecules, such as full triaxial ellipsoids, a different symmetry will ensue with a different realization of orientational degeneracy.

For any orthogonal transformation $\mathbf{U}$, an explicit calculation shows that

$$
\frac{\partial}{\partial \mathbf{m}} f(\mathbf{U} \cdot \mathbf{m}, t)=\mathbf{U}^{T} \cdot \frac{\partial}{\partial \mathbf{n}} f(\mathbf{n}, t)
$$

where

$$
\mathbf{n}=\mathbf{U} \cdot \mathbf{m}
$$

It then follows that

$$
\frac{\partial}{\partial \mathbf{m}} \cdot \frac{\partial}{\partial \mathbf{m}} f(\mathbf{U} \cdot \mathbf{m}, t)=\frac{\partial}{\partial \mathbf{n}} \cdot \frac{\partial}{\partial \mathbf{n}} f(\mathbf{n}, t)
$$

With $f_{U}(\mathbf{m}, t)=f(\mathbf{U} \cdot \mathbf{m}, t)$, the corresponding intermolecular potential is given by

$$
\begin{aligned}
& V\left(\mathbf{m}, f_{U}(\cdot, t)\right)=\int_{\left\|\mathbf{m}^{\prime}\right\|=1} h\left(\mathbf{m}, \mathbf{m}^{\prime}\right) f\left(\mathbf{U} \cdot \mathbf{m}^{\prime}, t\right) d \mathbf{m}^{\prime} \\
& =\int_{\left\|\mathbf{n}^{\prime}\right\|=1} h\left(\mathbf{m}, \mathbf{U}^{T} \cdot \mathbf{n}^{\prime}\right) f\left(\mathbf{n}^{\prime}, t\right) d \mathbf{n}^{\prime}=\int_{\left\|\mathbf{n}^{\prime}\right\|=1} h\left(\mathbf{U} \cdot \mathbf{m}, \mathbf{n}^{\prime}\right) f\left(\mathbf{n}^{\prime}, t\right) d \mathbf{n}^{\prime} \\
& =V(\mathbf{n}, f(\cdot, t)) .
\end{aligned}
$$

It follows that

$$
\begin{aligned}
& \frac{\partial}{\partial \mathbf{m}} \cdot \frac{\partial}{\partial \mathbf{m}} V\left(\mathbf{m}, f_{U}(\cdot, t)\right)=\frac{\partial}{\partial \mathbf{n}} \cdot \frac{\partial}{\partial \mathbf{n}} V(\mathbf{n}, f(\cdot, t)), \\
& \frac{\partial}{\partial \mathbf{m}} f\left(\mathbf{U} \cdot \mathbf{m}^{\prime}, t\right) \cdot \frac{\partial}{\partial \mathbf{m}} V\left(\mathbf{U} \cdot \mathbf{m}, f_{U}(\cdot, t)\right)=\frac{\partial}{\partial \mathbf{n}} f(\mathbf{n}, t) \cdot \frac{\partial}{\partial \mathbf{n}} V(\mathbf{n}, f(\cdot, t)) .
\end{aligned}
$$


Combining $(15,16,17)$, we have

$$
\begin{aligned}
& \frac{\partial}{\partial t} f_{U}(\mathbf{m}, t)=D_{r}(a) \mathcal{R} \cdot\left[\left(\mathcal{R} f_{U}+\frac{1}{k T} f_{U} \mathcal{R} V\left(\mathbf{m}, f_{U}(\cdot, t)\right)\right]\right. \\
& =D_{r}(a) \frac{\partial}{\partial \mathbf{m}} \cdot\left[\frac{\partial}{\partial \mathbf{m}} f_{U}+\frac{1}{k T} f_{U} \frac{\partial}{\partial \mathbf{m}} V\left(\mathbf{m}, f_{U}(\cdot, t)\right)\right]
\end{aligned}
$$

or equivalently,

$$
\begin{aligned}
& \frac{\partial}{\partial t} f(\mathbf{n}, t)=D_{r}(a) \frac{\partial}{\partial \mathbf{n}} \cdot\left[\frac{\partial}{\partial \mathbf{n}} f+\frac{1}{k T} f \frac{\partial}{\partial \mathbf{n}} V(\mathbf{n}, f(\cdot, t))\right] \\
& =D_{r}(a) \mathcal{R}_{\mathbf{n}} \cdot\left[\left(\mathcal{R}_{\mathbf{n}} f+\frac{1}{k T} f \mathcal{R}_{\mathbf{n}} V(\mathbf{n}, f(\cdot, t))\right]\right.
\end{aligned}
$$

where $\mathcal{R}_{\mathbf{n}}=\mathbf{n} \times \frac{\partial}{\partial \mathbf{n}}$. Thus, if $f(\mathbf{m}, t)$ satisfies the Smoluchowski equation, so does $f_{U}(\mathbf{m}, t)$. We can now restate the:

Constructive Form of Orientational Degeneracy. Every anisotropic solution $f(\mathbf{m}, t)$ of the Smoluchowski equation for nematic liquids at rest(8), for arbitrary initial data $f(\mathbf{m}, t=$ $0) \neq \frac{1}{4 \pi}$, generates an entire $O(3)$ group of solutions, $f(\mathbf{U} \cdot \mathbf{m}, t)$, for arbitrary $\mathbf{U} \in O(3)$. The isotropic solution $\frac{1}{4 \pi}$ is a fixed point of the symmetry, giving the lone isolated, nondegenerate, solution.

There are several consequences of this result.

- The set of equilibrium solutions of (8) versus nematic concentration $N$ comprises the isotropic-to-nematic phase transition diagram; the two anisotropic solution branches $f_{+}(\mathbf{m}), f_{-}(\mathbf{m})$ each correspond to a continuous group of equilibria parametrized by all elements $U \in O(3), f_{+}(\mathbf{U} \cdot \mathbf{m}), f_{-}(\mathbf{U} \cdot \mathbf{m})$.

Figure 1 gives the standard diagram using our own code [9], in agreement with [7, 11]. Solutions are, following tradition [15, 16], characterized and visualized in terms of the projection of $f$ onto the second-moment tensor $\mathbf{M}$, or its traceless form $\mathbf{Q}$. This allows one to make contact with the Landau-deGennes, Beris-Edwards [1], and Doi closure models [6] for the mesoscopic orientation tensor Q. Figure 2 illustrates two color-coded equilibrium distributions $f$ on the sphere, each corresponding to the stable nematic branch $f^{+}$of Figure 1 for nematic concentration $N=5$. Figure $2 \mathrm{a}$ is the unique nematic equilibrium distribution for $N=5$ with major director aligned with the z-axis; note the uniaxial degeneracy of quiescent equilibria which implies isotropy in all planes orthogonal to the major director axis (here $z=$ constant). Figure $2 \mathrm{~b}$ gives the transformed equilibrium distribution $f(\mathbf{U} \cdot \mathbf{m})$, for the explicit choice

$$
\mathbf{U}=\left(\begin{array}{ccc}
\frac{\sqrt{3}}{3} & \frac{\sqrt{3}}{3} & \frac{\sqrt{3}}{3} \\
0 & \frac{\sqrt{2}}{2} & -\frac{\sqrt{2}}{2} \\
-\frac{2 \sqrt{6}}{6} & \frac{\sqrt{6}}{6} & \frac{\sqrt{6}}{6}
\end{array}\right)
$$




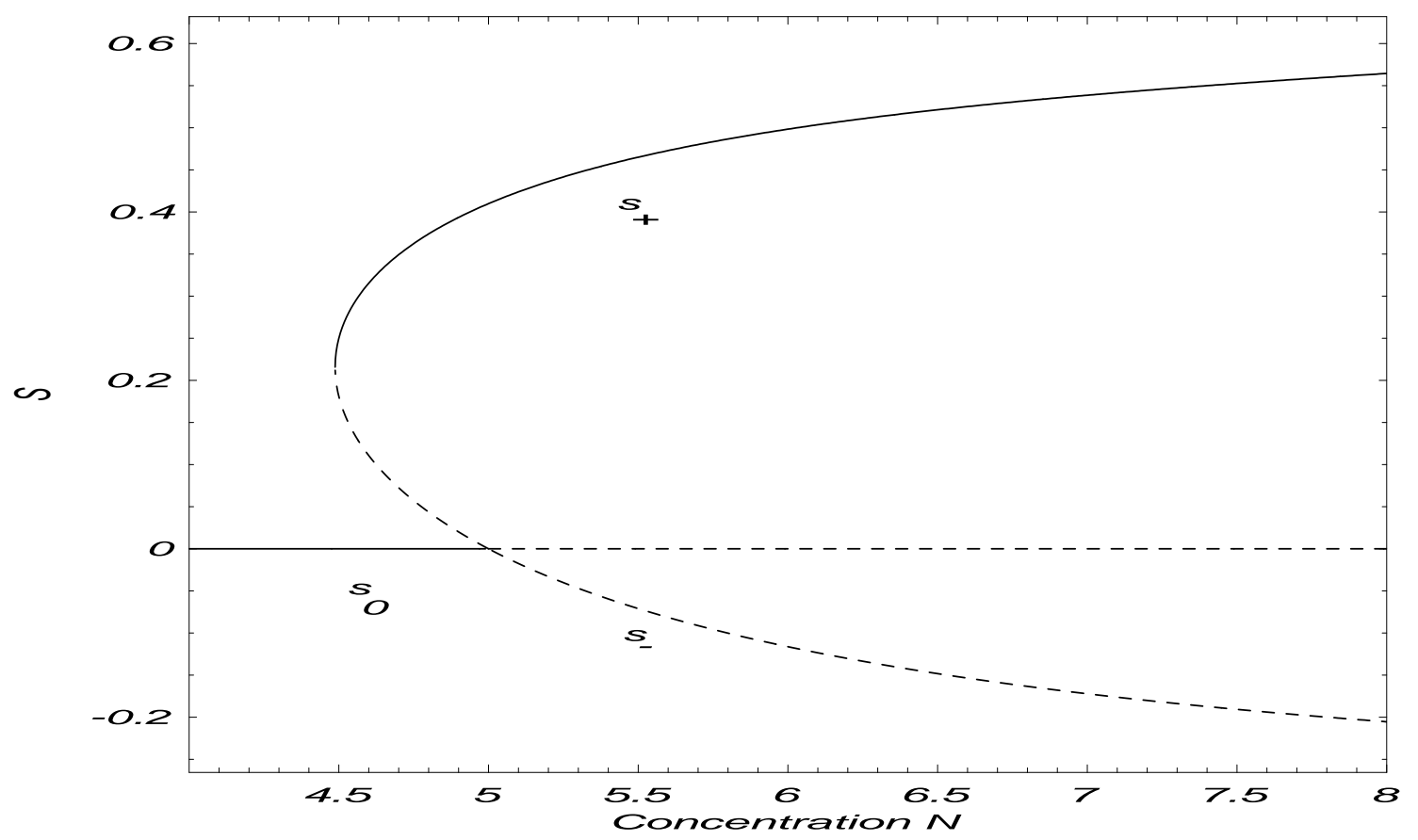

Figure 1: The isotropic-to-nematic phase diagram for quiescent nematic liquids of infinite aspect ratio. The curves depict equilibria $f_{*}$ of the Smoluchowski equation (8) versus dimensionless nematic concentration, $N$, for the Maier-Saupe intermolecular potential which has all uniaxial equilibria. The vertical axis depicts the traditional uniaxial order parameter, $s_{*}$, derived from the projection of $f_{*}$ onto the second-moment tensor $\mathbf{Q}_{*}$, from which $s_{*}$ is the simple eigenvalue. Solid lines represent stable $\left(s_{0}\right.$ for low $\left.N\right)$ or center-stable $\left(s_{+}\right.$for sufficiently large $N$ ) steady solutions; dashed lines correspond to unstable steady solutions. $f_{0}=\frac{1}{4 \pi}$ is the isotropic state, whose second-moment tensor $\mathbf{Q}_{0}=\mathbf{0}$ has triple eigenvalue $s_{0}=0$; this equilibrium is isolated, and non-degenerate. The nematic branches $f_{+,-}$correspond to an entire $O(3)$ of equilibria of the form $f_{+,-}(\mathbf{U} \cdot \mathbf{m})$ for all $\mathbf{U} \in O(3)$. The projections of $f_{+,-}$onto second-moment tensors, $\mathbf{Q}_{+,-}$, are likewise explicitly given by $\mathbf{U}^{T} \cdot \mathbf{Q}_{+,-} \cdot \mathbf{U}$. It then follows that the order parameter projections are unique for each entire group of equilibria: orthogonal similarity transformations preserve eigenvalues while moving the uniaxial director to another location on the sphere, parametrized by $\mathbf{U} \in O(3)$. 
(a)

(b)

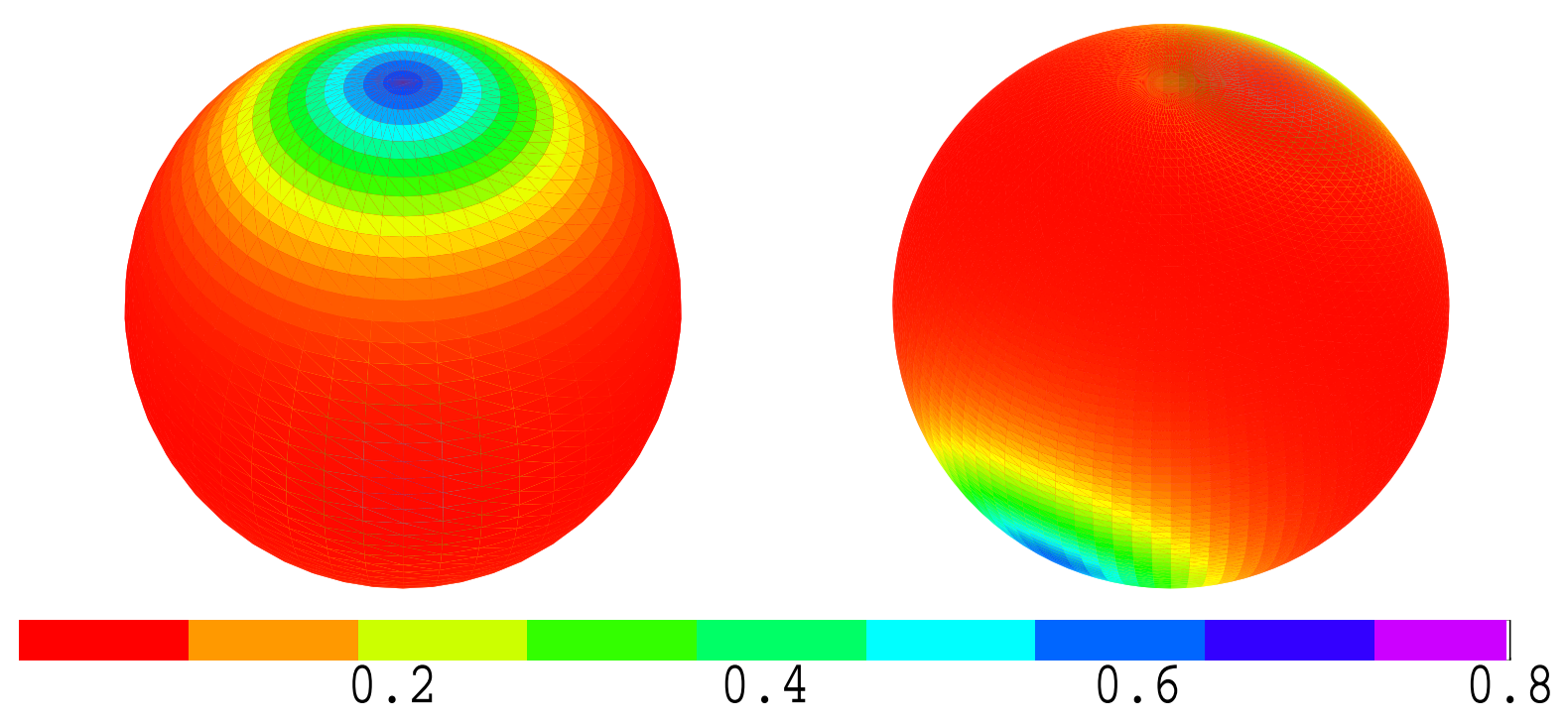

Figure 2: Illustration of orientational degeneracy for the nematic equilibrium $f_{+}$ at fixed concentration $N=5$. The left figure shows the unique nematic equilibrium distribution $f^{+}(\mathbf{m})$ specified by positing the major director aligns with the $z$-axis (pole axis). The right figure corresponds to another element of the group of equilibria, $f^{+}(\mathbf{U} \cdot \mathbf{m})$, for the specific element $\mathbf{U} \in O(3)$ given in (20). 


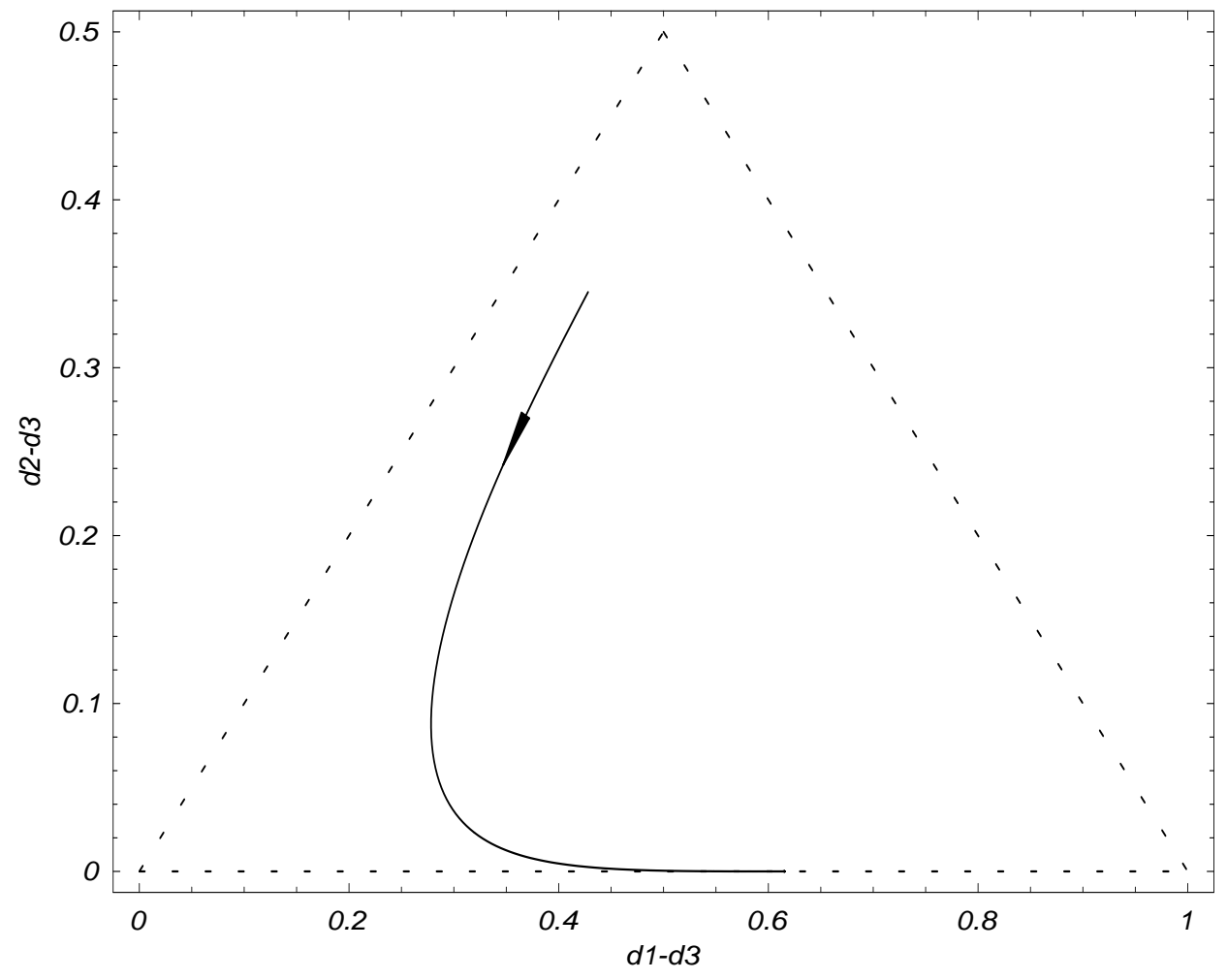

Figure 3: Orientational symmetry of orbits in the stable manifold of the nematic equilibrium $f^{+}$. Fix $N=5$ as in Figure 2. Two orbits $f_{1,2}(\mathbf{m}, t)$ of the Smoluchowski equation without flow (8) are numerically computed; the initial data $f_{1}(\mathbf{m}, 0)$ is in-plane, defined by (32) for the $\mathrm{x}-\mathrm{y}$ plane, whereas $f_{2}(\mathbf{m}, 0)=f_{1}(\mathbf{U} \cdot \mathbf{m}, 0)$ is an out-of-plane rotation of $f_{1}$ for $\mathbf{U}$ given in (20). These orbits converge to the equilibrium distributions imaged in Figure 2, and orientational symmetry implies $f_{2}(\mathbf{m}, t)=f_{1}(\mathbf{U} \cdot \mathbf{m}, t)$ for all time, which we do not enforce but rather confirm by numerical integration. Each orbit is projected onto the second-moment tensor $\mathbf{M}_{1,2}(t)$, which according to orientational symmetry must be related by a similarity transformation by $\mathbf{U}(20), \mathbf{M}_{2}(t)=\mathbf{U}^{T} \cdot \mathbf{M}_{1}(t) \cdot \mathbf{U}$. Since eigenvalues are invariant under orthogonal similarity transformations, we can illustrate the symmetry by showing the eigenvalues of each second-moment are identical during the entire evolution to the uniaxial equilibria of Figure 2. The eigenvalues are ordered $1 \geq d_{1} \geq d_{2} \geq d_{3} \geq 0$. Indeed the curves lie on top of one another, and converge to the uniaxial distributions with $d_{2}=d_{3}$. 
We emphasize these symmetries give a priori control of the "most probable directions" of orientation in Figures 2a,b, which coincide with the "major director" defined in terms of the second-moment Q-tensor projection of $f$. That is, if two pdf $f_{1,2}$ are related by the symmetry $f_{1}(\mathbf{m})=f_{2}(\mathbf{U} \cdot \mathbf{m})$, then their second moment projections $\mathbf{Q}_{1,2}$ automatically satisfy $\mathbf{Q}_{1}=$ $\mathbf{U}^{T} \cdot \mathbf{Q}_{2} \cdot \mathbf{U}$, which reproduces the mesoscopic form of the symmetry [8]. In numerical simulations, a random initial distribution $f(\mathbf{m}, t=0)$ might converge numerically to Figure 2b for $N=5$, from which one can deduce the Euler angles of the "major director". But the "direct problem", where one prescribes the Euler angles of the equilibrium distribution in Figure 2b, is only solved with symmetries. We a priori parametrize all data for (8) that converge to the distributions of Figure $2 \mathrm{a}, 2 \mathrm{~b}$, or any orthogonal rotation of these spherical distributions, the union of which comprise $f^{+}$for $N=5$ in Figure 1.

The construction proceeds as follows. We first borrow a result from the next section (32), which characterizes "in-plane orientation" at the kinetic equation level of the pdf $f$, and which preserves the "in-plane" definition based on the Q-tensor projection of $f$. This symmetry allows us to initialize random in-plane, biaxial initial distributions $f_{i p}(\mathbf{m}, 0)$; by the group symmetry, every such orbit is guaranteed to remain in-plane, and therefore (from stability results for $N=5$, Figure 1) all such anisotropic initial data are in the stable manifold of an in-plane nematic equilibrium $f_{i p}^{+}(\mathbf{m})$ (Figure 2a). We have chosen biaxial initial data for Figure 3 whose major director aligns with the $z$-axis, and therefore the distribution converges to the uniaxial in-plane distribution of Figure 2a. Figure 3a plots the projection of this orbit onto the order parameters of the Q-tensor (equivalently, $\mathbf{M}$ ); the directors are not shown, since they are passive during the evolution. Next we rotate the initial data out-of-plane, $f_{\text {op }}(\mathbf{m}, 0)=f_{i p}(\mathbf{U} \cdot \mathbf{m}, 0)$ with the specific choice $(20)$. The numerical integration of this data, by the symmetry, has to agree with the U-transformation of the in-plane pdf solution. Therefore, the orbit is guaranteed a priori to converge to the distribution given in Figure $2 \mathrm{~b}$ ! This is confirmed numerically, and offers a good benchmark on the code and visualization routines. Since $f_{o p}$ and $f_{i p}$ have $\mathbf{Q}$ tensor projections related by $\mathbf{Q}_{o p}(t)=\mathbf{U}^{T} \cdot \mathbf{Q}_{i p} \cdot \mathbf{U}(t)$ for all time, we are guaranteed that the eigenvectors are initially transformed by $\mathbf{U}$ and do not vary in time, whereas the eigenvalues (order parameters) of each Q-tensor evolve identically for all time (Figure 3).

We now recall essential ingredients of Galerkin expansions for the Smoluchowski equation and the projection formula for the second moment tensor. The spherical harmonic expansion for $f[15,16,7,11]$ is:

$$
f(\mathbf{m}, t) \approx \sum_{l=0}^{L} \sum_{m=-l}^{l} a_{l, m}(t) Y_{l}^{m}(\theta, \phi),
$$


where $Y_{l}^{m}$ are complex spherical harmonic basis functions [25],

$$
Y_{l}^{m}=P_{l}^{m}(\cos \theta) e^{i m \phi},
$$

$P_{l}^{m}$ are Legendre polynomials, $\theta, \phi$ are spherical coordinates with $\theta$ the polar angle and $\phi$ the latitude angle, and $L$ is the order of truncation in the Galerkin approximation.

In the Appendix, we give an explicit form (47) of the dynamical system for the amplitudes $a_{l, m}(t)$ which results from the above expansion for $f$ in the Smoluchowski equation with and without an imposed linear flow field. These equations are not new; this form is used to prove the flow-nematic kinetic symmetries.

The three amplitudes $a_{2,0}, a_{2,1}, a_{2,2}$ uniquely specify the second-moment tensor $\mathbf{Q}$ :

$$
\begin{aligned}
& Q_{x x}=-\frac{2}{3} \sqrt{\frac{\pi}{5}} a_{2,0}+\sqrt{\frac{8 \pi}{15}} \Re\left(a_{2,2}\right) \\
& Q_{y y}=-\frac{2}{3} \sqrt{\frac{\pi}{5}} a_{2,0}-\sqrt{\frac{8 \pi}{15}} \Re\left(a_{2,2}\right) \\
& Q_{x y}=\sqrt{\frac{8 \pi}{15}} \Im\left(a_{2,2}\right) \\
& Q_{x z}=-\sqrt{\frac{8 \pi}{15}} \Re\left(a_{2,1}\right) \\
& Q_{y z}=-\sqrt{\frac{8 \pi}{15}} \Im\left(a_{2,1}\right)
\end{aligned}
$$

where, $\Re(\cdot)$ and $\Im(\cdot)$ represent the real and the imaginary part, respectively.

The amplitudes $a_{l, m}(t)$ of $f$ are equivalent to the moments of the distribution $f$; the only way to recover a continuous (without flow) or discrete (with flow) symmetry from the moment equations is through a transformation on the space of solutions which maps orbits of the dynamical system $a_{l, m}(t)$ onto new orbits. All discrete flow symmetries are described below in this way.

\section{Mirror-symmetry of all out-of-plane responses to sim- ple shear}

In this section we specialize the linear velocity field in (1) to simple shear with arbitrary shear rate $\dot{\gamma}$, represented in standard Cartesian coordinates:

$$
\mathbf{v}=\dot{\gamma}(y, 0,0)
$$

In [8], we prove that the pre-closure form of the mesoscopic Doi theory admits a reflection symmetry: any orbit $\mathbf{Q}(t)$ of the mesoscopic theory generates another orbit symmetric about 
the shearing plane. A constructive algorithm satisfied by all solutions is given: retain the same values of in-plane components, $Q_{x x}, Q_{x y}, Q_{y y}, Q_{z z}$, and reverse the sign of both outof-plane components, $Q_{x z}, Q_{y z}$. Any out-of-plane solution will generate a solution which is distinct pointwise in time; if the entire orbit is not symmetric with respect to the vorticity axis, then the solutions are distinct. Logrolling and classical kayaking orbits which rotate around the vorticity axis are mapped to themselves, and therefore are unique. This discrete reflection symmetry is compactly represented in terms of a similarity transformation of the mesoscopic tensor $\mathbf{Q}(t)$, employing the planar reflection transformation $\mathbf{V}_{2}$ :

$$
\mathbf{V}_{2}^{T} \cdot \mathbf{Q}(t) \cdot \mathbf{V}_{2}
$$

where the Cartesian representation of reflection through the $(\mathrm{x}, \mathrm{y})$ plane is

$$
\mathbf{V}_{2}=\operatorname{diag}(1,1,-1)
$$

These two formulations of shear-induced mirror symmetry are now generalized to the Smoluchowski equation (1) with imposed shear flow (28). First we give the constructive version in terms of a discrete transformation on the spherical harmonic amplitudes $a_{l, m}$. Reflection symmetry in terms of the spherical harmonic expansion of the orientational distribution function $f$. Every solution $f(\mathbf{m}, t)$ of $(1)$ has spherical harmonic amplitudes $a_{l, m}(t)$, which generate another pdf solution $f^{\text {sym }}(\mathbf{m}, t)$ with amplitudes

$$
(-1)^{m} a_{l, m}(t)
$$

Moreover, the orientational distributions $f$ and $f^{s y m}$ are mirror-symmetric with respect to the shearing plane, generalizing the mesoscopic symmetry (29).

In-plane configurations remain in-plane for all time. We define an in-plane subspace of orientational distribution functions by the infinite set of conditions which generalize the Q-tensor in-plane conditions $Q_{x z}=Q_{y z}=0$ :

$$
a_{l, m}=0, \text { for all } m \text { odd. }
$$

The entire in-plane subspace is invariant under the flow of the Smoluchowski equation (1). I.e., all in-plane orbits $f(\mathbf{m}, t)$ are mapped to themself by this symmetry. This implies that in-plane orientational configurations cannot become out-of-plane, nor can any out-of plane configurations become in-plane, in finite time. Out-of-plane orbits whose major director does not align with the vorticity axis nor rotate about the vorticity axis must remain "tilted" to one side of the shearing plane. Explicit examples include branches of out-of-plane steady and periodic states identified in [7], which they explicitly recognized to occur in mirror-symmetric 

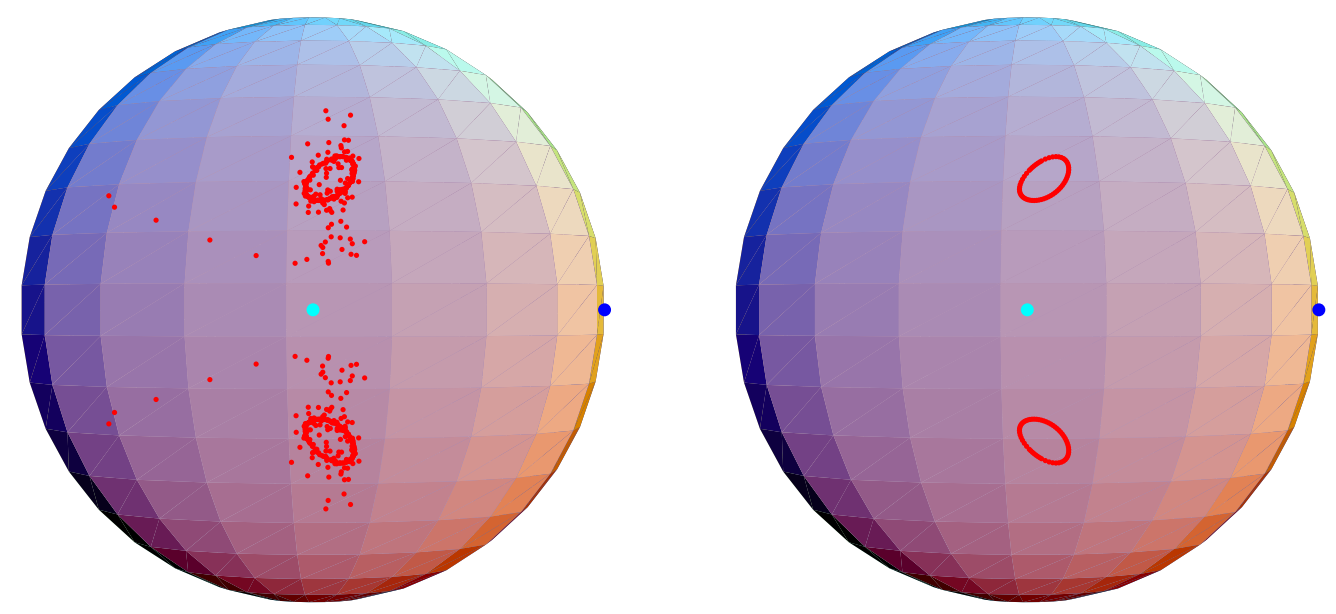

Figure 4: Reflection-symmetric, out-of-plane, periodic solutions of the shear-driven Smoluchowski equation (1) for infinite-aspect-ratio, rod-like macromolecules $(a=1)$, nematic concentration $N=5.5$, and normalized shear rate $P e=6$. The primary director of the Q-tensor projection of $f(\mathbf{m}, t)$ is shown for each attracting monodomain state, illustrating discrete mirror symmetry with respect to the shearing plane. The equator corresponds to the shear plane; the blue dot represents the flow direction $(x)$ and the black dot represents the flow gradient direction $(y)$. These symmetric attractors, reported in [7], may be viewed as either "tilted kayaking orbits" or "out-of-plane wagging orbits". The right figure shows the attractor. The left figure shows the orbit that converges to this attractor from an arbitrarily chosen initial data. The data is recorded from every 15 th time step. 
pairs. This symmetry explains their observations, but also implies every orbit in the stable and unstable manifolds of every solution, in-plane or out-of-plane, has a mirror-symmetric orbit. Figure 4 illustrates the reflection-symmetry in simple shear. Figure 4a illustrates two mirror-symmetric orbits for a fixed shear rate and nematic concentration; the equator in this figure is the shear plane, the north pole is the vorticity axis. The orbit in the northern hemisphere converges to a "tilted, out-of-plane periodic" attractor, shown in Figure 4b. Each orbit and attractor are mirrored by another numerical solution generated by reflecting the initial data through the shearing plane. Here, the pdf $f$ is projected onto the second-moment tensor, from which the major director is extracted at each time step.

Other consequences for dynamics of monodomains follow. For example, if a tumbling or wagging in-plane orbit is the unique attractor, then out-of-plane orbits converge symmetrically from both sides of the shearing plane; a similar picture occurs for convergence to steady in-plane attractors. Likewise, many in-plane solutions are unstable to out-of-plane perturbations; their unstable manifolds are mirror-symmetric. We note the pdf solutions identified with the logrolling steady state and the classical Larson-Ottinger kayaking orbit are mapped onto themself by this symmetry; therefore such monodomain attractors are isolated and do not occur in pairs.

Another proof of the mirror-reflection symmetry (31) is gained by fixing the coordinate representation of the pdf $f$ in the Smoluchowski equation (1), then applying the reflection transformation, for which the equation remains invariant. We write this result in terms of any finite aspect ratio fluid with molecular geometry parameter $a$, a fixed shear flow (28), and a corresponding solution $f$ of the kinetic equation (1). We note:

$$
\mathbf{V}_{2} \cdot \mathbf{v}_{\text {shear }}=\mathbf{v}_{\text {shear }}, \quad \mathbf{V}_{2} \cdot \Omega \cdot \mathbf{V}_{2}^{T}=\Omega, \quad \mathbf{V}_{2} \cdot \mathbf{D} \cdot \mathbf{V}_{2}^{T}=\mathbf{D} \text {. }
$$

So, the time evolution of $\mathbf{n}=\mathbf{V}_{2} \cdot \mathbf{m}$ obeys (2) and

$$
\begin{aligned}
& \mathcal{R} \cdot\left(\mathbf{m} \times \dot{\mathbf{m}} f_{\mathbf{V}_{2}}(\mathbf{m}, t)\right)=\frac{\partial}{\partial \mathbf{m}} \cdot\left(\dot{\mathbf{m}} f_{\mathbf{V}_{2}}(\mathbf{m}, t)\right) \\
& =\frac{\partial}{\partial \mathbf{n}} \cdot(\dot{\mathbf{n}} f(\mathbf{n}, t))=\mathcal{R}_{\mathbf{n}} \cdot(\mathbf{n} \times \dot{\mathbf{n}} f(\mathbf{n}, t)) .
\end{aligned}
$$

The flow-independent part follows from our proof of the orientational degeneracy in equilibrium. Thus, we have established a transformation between solutions of the two Smoluchowski equations defined by the triples,

$$
\left(a, \mathbf{v}_{\text {shear }}, f\right), \quad\left(a, \mathbf{V}_{2} \cdot \mathbf{v}_{\text {shear }}, f_{\mathbf{V}_{2}}\right) .
$$




\section{Orientational equivalence between rod-like and dis- cotic nematic fluids in simple shear, for finite and infinite aspect ratios}

We now establish a remarkable equivalence in the shear response of nematic liquids with reciprocal aspect ratios, $r$ and $\frac{1}{r}$. This result was previously established in [8] for tensor models. The $\mathbf{Q}_{r}$-tensor (the subscript labels aspect ratio $r$ ) response to simple shear at fixed nematic concentration $N$ for rods $(r>1)$ generates the orientational response at the same shear rate and concentration for a discotic liquid of aspect ratio $\frac{1}{r}$. Indeed they are related by the explicit transformation

$$
\mathbf{Q}_{r^{-1}}=\mathbf{V}_{ \pm}^{T} \cdot \mathbf{Q}_{r} \cdot \mathbf{V}_{ \pm}
$$

where $\mathbf{V}_{ \pm}$is a pure clockwise, respectively counterclockwise, rotation by $\frac{\pi}{2}$ radians in the shearing plane which fixes the vorticity axis:

$$
\mathbf{V}_{+}=\left(\begin{array}{ccc}
0 & 1 & 0 \\
-1 & 0 & 0 \\
0 & 0 & 1
\end{array}\right), \quad \mathbf{V}_{-}=\mathbf{V}_{+}^{T}
$$

All orientational responses of rod-like and discotic liquids in simple shear are related by this $1: 1$ correspondence. We now show this is simply the $\mathbf{Q}$-tensor projection of a general kinetic theory symmetry. We first give a constructive correspondence in terms of the spherical harmonic expansion of $f$, and then a general statement independent of the representation of $f$.

Recall the molecular geometry parameter in (1), $a=\frac{r^{2}-1}{r^{2}+1}$, where $r$ is the molecular aspect ratio. Thus, $r$ and $\frac{1}{r}$ correspond to $a$ and $-a$, which appears parametrically in the Smoluchowski equation (1).

Rod-discotic correspondence in simple shear. Fix any shear rate $\dot{\gamma}$ and nematic concentration $N$; rod-like (discotic) liquids are parametrized by positive (negative) aspect ratio parameter $|a|(-|a|)$. Each solution $f_{|a|}(\mathbf{m}, t)$ of the Smoluchowski equation (1) for arbitrary initial data $f(\mathbf{m}, t=0)$ has the spherical harmonic expansion (21) with a unique set of amplitude functions $a_{l, m}^{+}(t)$. Transform the initial data and corresponding pdf solution as follows:

$$
\begin{aligned}
& a_{l, m}^{-}(0)=i^{m} a_{l, m}^{+}(0), \quad i=\sqrt{-1}, \\
& a_{l, m}^{-}(t)=i^{m} a_{l, m}^{+}(t) .
\end{aligned}
$$


From these data, construct the distribution function $f_{-|a|}(\mathbf{m}, t)$ by the spherical harmonic expansion (21). Then, $f_{-|a|}$ is a solution of (1) for fluids with aspect ratio parameter $-|a|$ and initial data $f(\mathbf{m}, 0)$ defined by $a_{l, m}^{-}(0)$.

To prove that this symmetry maps all solutions of (1) for aspect ratio $r$ to solutions for aspect ratio $\frac{1}{r}$, it is a routine calculation to verify the following symmetry of the dynamical system for the amplitudes $a_{l, m}$ given in the Appendix. With $i=\sqrt{-1}$,

$$
F_{l, m}^{j}=i^{m} F_{l, m}^{j}, \quad l=2,4, \cdots, \quad-l \leq m \leq l
$$

for $j=1,2,3$ under the above transform (38). We remark that the corresponding result for mesoscopic tensor models [8] is far easier to discover and prove, from which this symmetry was found.

If one iterates this transformation, then individual solutions $f(\mathbf{m}, t)$ are mapped to themself if they are in-plane, but to their mirror-symmetry through the shear plane if they are out-of-plane.

In Figure 5 we illustrate this symmetry by plotting all solution branches, stable and unstable, of the Smoluchowski equation (1) for all aspect ratio parameters $a \in[-1,+1]$, at a particular fixed concentration $N$ and fixed shear rate $P e$. We graph the projection of all solutions $f$ onto second-moment Q-tensor components, as noted in the caption. Note the above symmetry implies $a_{2,0}$, which is proportional to the in-plane component $Q_{z z}$, is an even function of $a ; \Re\left(a_{2,2}\right)$, which is proportional to the in-plane component $Q_{x x}-Q_{y y}$, is an odd function of $a$; the out-of-plane components, $\Re\left(a_{2,1}\right)$ and $\Im\left(a_{2,1}\right)$, which are proportional to $Q_{x z}$ and $Q_{y z}$, respectively, are mapped onto one another by the symmetry, i.e., $Q_{x z}(-a)=Q_{y z}(a)$ ! These symmetries are evident in Figure 5. Since periodic solutions are represented by a pointwise statistic (maximum value of the indicated component over one period), the odd symmetry of $\Re\left(a_{22}\right)$ is not evident for the periodic branches in Figure 5b.

There is another formulation of the rod-discotic symmetry. A one-to-one correspondence exists between the orientational distribution functions $f$ for two nematic liquids of aspect ratio parameters $a$ and $-a$. This transformation can be formulated in the following way. The Smoluchowski equation (1) is uniquely specified by a triple, $(a, \mathbf{v}, f)$, where $a$ is the molecular geometry parameter, $\mathbf{v}$ is a linear flow, and $f$ is the pdf for fixed $N, P e$. Let

$$
\mathbf{n}=\mathbf{V}_{ \pm} \cdot \mathbf{m}
$$

We note the following properties of the rotational transformations:

$$
\mathbf{V}_{ \pm} \cdot \Omega \cdot \mathbf{V}_{ \pm}^{T}=\Omega, \quad \mathbf{V}_{ \pm} \cdot \mathbf{D} \cdot \mathbf{V}_{ \pm}^{T}=-\mathbf{D}
$$

It then follows from (2) that for simple shear (28)

$$
\dot{\mathbf{n}}=\Omega \cdot \mathbf{n}-a[\mathbf{D} \cdot \mathbf{n}-\mathbf{D}: \mathbf{n n n}],
$$



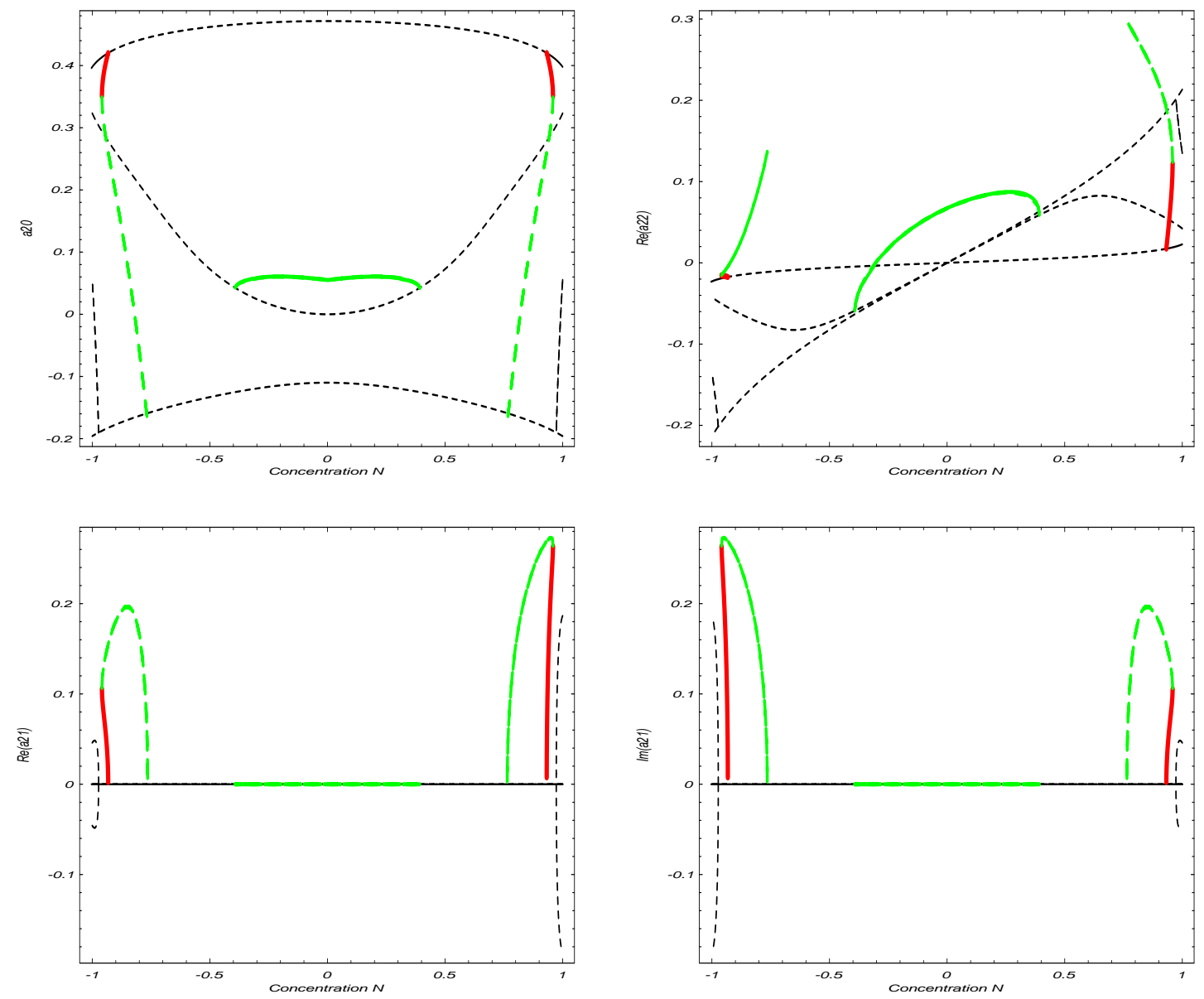

Figure 5: Illustration of the rod-discotic symmetry of monodomain orientational distributions for nematic liquids in simple shear. All stable and unstable solution branches are depicted for $a \in[-1,1]$, equivalently $r \in[-\infty,+\infty]$, for the Smoluchowski equation (1) with fixed nematic concentration $N=6$, and relatively strong n normalized shear rate $P e=8.5$. Four spherical harmonic amplitude components are plotted, which correspond to projection of the orientational distribution $f$ onto the second-moment tensor, as seen from (27): the in-plane components $a_{2,0}$ (proportional to $Q_{z z}$ ) and $\Re\left(a_{2,2}\right)$ (proportional to $\left.Q_{x x}-Q_{y y}\right)$, and the out-of-plane components $\Re\left(a_{2,1}\right)$ (proportional to $\left.Q_{x z}\right)$ and $\Im\left(a_{2,1}\right)$ (proportional to $Q_{y z}$ ). The solid thin line indicates a stable steady solution; the dashed line indicates an unstable steady solution branch; the red (solid, dark) line indicates stable periodic solutions; the blue (grey) line indicates unstable periodic solutions. For periodic solutions, the maximum value of the indicated quantity is given, which breaks the perfect odd symmetry of $\Re\left(a_{22}\right)$ in the top right graph. The figures illustrate: $a_{20}$ is an even function of $a ; \Re\left(a_{22}\right)$ is an odd function of $a$; and $\Re\left(a_{21}\right)$ for $a$ maps onto $\Im\left(a_{21}\right)$ for $-a$. 
so the Jeffery molecular orbit equation for $\mathbf{n}$ is recovered if we replace $a$ by $-a$, i.e., the Jeffery orbit is invariant under the transformation $(a, \mathbf{m}) \rightarrow\left(-a, \mathbf{n}=\mathbf{V}_{ \pm} \cdot \mathbf{m}\right)$. The result follows from $(15,16,17,34)$. Thus, we establish the 1:1 correspondence between the Smoluchowski equations, and therefore between all solutions:

$$
\left(a, \mathbf{v}_{\text {shear }}, f\right) \rightarrow\left(-a, \mathbf{V}_{ \pm} \cdot \mathbf{v}_{\text {shear }}, f_{\mathbf{v}_{ \pm}}\right)
$$

As illustrated in Figure 5, all phase transitions (bifurcations versus $N$ or $P e$ ), all stable and unstable, steady or transient, monodomain states occur simultaneously for rods and discotics of reciprocal aspect ratios. We caution that the constitutive stress equation does not preserve this symmetry, since Miesowicz viscosities and elasticity constants vary greatly between rod-like and discotic nematic polymers [32]. Therefore, this 1:1 correspondence is for the orientational, homogeneous response to imposed shear. The rheological response, and subsequent structure formation, do not share this correspondence.

\section{A one-to-one correspondence between finite and in- finite aspect-ratio fluids in related linear flows}

We again note the Smoluchowski equation (1) defines a "triple": $(a, \mathbf{v}, f(\mathbf{m}, t))$. Consider an arbitrary linear flow given by

$$
\mathbf{v}=(\boldsymbol{\Omega}+\mathbf{D}) \cdot \mathbf{x}
$$

We observe from (1) that the rate-of-strain tensor $\mathbf{D}$ and geometry parameter $a$ enter linearly and only through their product. This fact underlies two symmetries of the system (1) which we describe in terms of the triple defined above:

$$
\begin{aligned}
& (a, \mathbf{v}, f) \rightarrow(1,(\boldsymbol{\Omega}+a \mathbf{D}) \cdot \mathbf{x}, f) \\
& (a, \mathbf{v}, f) \rightarrow(-1,(\boldsymbol{\Omega}-a \mathbf{D}) \cdot \mathbf{x}, f)
\end{aligned}
$$

These symmetries imply an identical monodomain response $f$ of: a finite aspect ratio fluid with geometry parameter $a$ in any linear flow field, and, extremely thin rodlike or discotic fluids, respectively, in a linear superposition of the identical linear flow field perturbed by a pure strain velocity component. This correspondence has experimental implications for monodomain behavior, as indicated in [8]. For example, the monodomain response to linear flow of an entire spectrum of monodisperse nematic liquids can be inferred from flow experiments on a single large-aspect-ratio nematic liquid by controlling the amplitude of the straining component while holding the vorticity component fixed. Alternatively, a finite-aspect-ratio, 
monodisperse nematic liquid in simple shear can be used to mimic more general linear flows of extremely thin rod-like or discotic fluids.

This symmetry provides physical intuition as to why molecular aspect ratio variations could lead to significant experimental changes relative to thin rod-like fluids. The change in aspect ratio, at least for the orientational distribution dynamics, corresponds to an equivalent experimental study where the planar shear flow is varied through a straining perturbation. In [8] we find dramatic changes in flow-phase diagrams when the molecular aspect ratio is reduced to the range of $5: 1$ to $3: 1$. Steady-unsteady transitions, new types of monodomains, and even a period-doubling transition to chaotic monodomain dynamics, result solely from aspect ratio variations. Corresponding sensitivity of kinetic flow-phase diagrams to molecular aspect ratio will be discussed elsewhere. We emphasize that these properties are restricted to linear flows and homogeneous orientational distributions, and do not imply such direct relationships for rheological behavior.

\section{Conclusion}

Several symmetries of the extended Doi kinetic theory for finite and infinite aspect ratio fluids in shear and related linear flows are now established, extending results from [8] for mesoscopic, moment-averaged approximations of the kinetic theory. These symmetries have been presented in abstract as well as in constructive form. Continuous families of orientational probability distributions $f$ for quiescent nematic liquids are characterized for every anisotropic initial orientational distribution. In simple shear, the pdf $f$ for out-of-plane initial data generates an explicit mirror-symmetric solution $f_{\mathbf{V}_{2}}$ with respect to the shearing plane. This symmetry explains previously reported bi-stable out-of-plane steady and periodic solutions [7], and further implies a mirror symmetry of all stable and unstable manifolds of in-plane and out-of-plane solution branches. Finally, orientational responses are shown to be in 1:1 correspondence for rod-like and discotic nematic liquids in simple shear, and a similar correspondence is provided between finite and infinite aspect ratio nematic liquids in linear flows related by a straining perturbation. All symmetries are illustrated with exact solutions or bifurcation diagrams, using codes developed in the sequel to this paper [9] where applications of these symmetries are pursued.

\section{Acknowledgment}

MGF and QW acknowledge partial financial support by AFOSR grants F49620-00-10008 and F49620-02-1-0086, respectively. The US Government is authorized to reproduce 
and distribute reprints for governmental purposes notwithstanding any copyright notation thereon. The views and conclusions contained herein are those of the authors and should not be interpreted as necessarily representing the official policies or endorsements, either expressed or implied, of the Air Force Office of Scientific Research or the US Government.

\section{References}

[1] Beris, A.N., Edwards, B.J., Thermodynamics of Flowing Systems with Internal Microstructure, Oxford Science Publications (1994).

[2] Bird, B., Armstrong, R.C., and Hassager, O., Dynamics of Polymeric Liquids, Volumes 1, 2, John Wiley and Sons (1987).

[3] Chillingworth, D.R.J., VicenteAlonso, E., Wheeler, A.A., J. Phys. A 34, 1393 (2001).

[4] deGennes, P.G., Prost, J., The Physics of Liquid Crystals, Oxford University Press, Oxford (1993).

[5] Doi, M. J. Polym. Sci., Polym. Phys. Ed. 19, 229 (1981).

[6] Doi, M. and Edwards, S. F., The Theory of Polymer Dynamics, Oxford U. Press (Clarendon), London-New York (1986).

[7] Faraoni, V., Grosso, M., Crescitelli, S., Maffettone, P. L., J. Rheol. 43, 829 (1999).

[8] Forest, M.G., Wang, Q., UNC Program in Applied Mathematics Preprint No. PAMPS2001-01, submitted to Rheol. Acta, October (2001).

[9] Forest, M.G., Wang, Q., Zhou, R., The effect of weak shear on the isotropic-nematic phase diagram for nematic polymers, UNC Program in Applied Mathematics Preprint No. PAMPS2002-06 (2002).

[10] Fuller, G.G., Optical Rheometry of Complex Fluids, Oxford University Press, Oxford (1995).

[11] Grosso, M., Keunings, R., Crescitelli, S., Maffettone, P.L., Prediction of chaotic dynamics in sheared liquid crystalline polymers, Preprint (2001) and lecture, Society of Rheology Annual Meeting, Hilton Head, SC, February (2001).

[12] Jeffery, G. B., Proc. Roy. Soc. London Ser. A 102, 161 (1922).

[13] Kuzuu, N., Doi, M.J., Phys. Soc. Japan 52, 3486 (1983).

[14] Kuzuu, N., Doi, M. J., Phys. Soc. Japan 53, 1031 (1984).

[15] Larson, R.G., Macromolecules 23, 3983 (1990).

[16] Larson, R.G., Ottinger, H., Macromolecules 24, 6270 (1991).

Liquid Crystals 12, 751 (1992).

[17] Maffettone, P.L., Crescitelli, S., J. Non-Newt. Fluid Mech. 59, 73 (1995). 
[18] Maffettone, P.L., Marrucci, G., Mortier, M. Moldenaers, P. Mewis, J. Chem. Phys. 100, 7736 (1994).

[19] Marrucci, G., Macromolecules 24, 4176 (1991).

[20] Marrucci, G., Greco, F., Mol. Cryst. Liq. Cryst. 206, 17 (1991).

[21] Marrucci, G., Greco, F., J. Non-Newt. Fluid Mech. 44, 1 (1992).

[22] Marrucci, G., Greco, F., Adv. Chem. Phys. 86, 331 (1993).

[23] Marrucci, G., Maffettone, P.L., Macromolecules 22, 4076 (1989).

[24] Marrucci, G., Maffettone, P.L., J. Rheol. 34, 1217, 1231 (1990).

[25] Messiah, Albert, Quantum Mechanics. Volume 2, North-Holland Publishing Company, Amsterdam, 1964

[26] Marsden, J. E., Ratiu, T. S., Introduction to mechanics and symmetry, SpringerVerlag, New York, 1994.

[27] Pradadarao, M. Pearce, E.M., Han, C.D., J. Appl. Polym. Sci., 27, 1343 (1982).

[28] See, H., Doi, M., Larson, R.G., J. Chem. Phy. 92(1), 792 (1990).

[29] Semenov, A.N., Sov. Phys. J.E.T.P. 85, 321 (1983).

[30] Semenov, A.N., Sov. Phys. J.E.T.P. 66, 712 (1986).

[31] Vicente Alonso, E., Wheeler, A.A., Sluckin, T.J., preprint of the University of Southampton, submitted (2002).

[32] Wang, Q., A hydrodynamic theory for solutions of nonhomogeneous nematic liquid crystalline polymers of different configuration, J. Chem Phys., to appear (2002).

Appendix The dynamical system for the amplitudes $\left\{a_{l, m}(t)\right\}$ in the spherical harmonic expansion of $f(21)$ results from a routine Galerkin procedure (cf., $[15,16,17])$ applied to the Smoluchowski equation (1) and Jeffery orbit dynamics (2). This system of odes can be represented in the form:

$$
\frac{d}{d t}\left(a_{l, m}\right)=F_{l, m}^{1}+F_{l, m}^{2}+F_{l, m}^{3}
$$

where

$$
\begin{gathered}
F_{l, m}^{1}=-D_{r}(\mathbf{m}, a) l(l+1) a_{l, m} . \\
F_{l, m}^{2}=\frac{4 \pi}{5} N(-1)^{m} D_{r}(\mathbf{m}, a) \sum_{p=-2}^{2} \sum_{n=l-2}^{l+2} a_{n, m-p} a_{2, p} q(l, m, n, p)
\end{gathered}
$$




$$
\begin{aligned}
F_{l, m}^{3}=i \frac{1}{4} \sqrt{\frac{8 \pi}{15}}(-1)^{m} \mu\left\{\sum_{n=l-2}^{l+2} a_{n, m} q_{1}(l, m, n)\right. & +a \sum_{n=l-2}^{l+2} a_{n, m+2} q_{2}(l, m, n) \\
& \left.+a \sum_{n=l-2}^{l+2} a_{n, m-2} q_{3}(l, m, n) .\right\}
\end{aligned}
$$

All numbers $q$ appearing above are real constants arising in spherical harmonic expansions:

$$
\begin{aligned}
q(l, m, n, p)= & -\frac{1}{2}\left[c_{l, m, 1} c_{2, p, 1}|n, m-p ; j, p+1 ; l,-m-1\rangle\right. \\
& \left.\quad+c_{l, m, 2} c_{2, p, 2}|n, m-p ; 2, p-1 ; l,-m+1\rangle\right] \\
& \quad+m p|n, m-p ; j, p ; l,-m\rangle \\
q_{1}(l, m, n)= & c_{l, m, 2}|l,-m+1 ; n, m ; 2,-1\rangle-c_{l, m, 1}|l,-m-1 ; n, m ; 2,1\rangle \\
& +2 m\left[\sqrt{\frac{10}{3}}|l,-m ; n, m ; 0,0\rangle-\sqrt{\frac{2}{3}}|l,-m ; m, k ; 2,0\rangle\right] \\
q_{2}(l, m, n)= & c_{l, m, 1}|l,-m-1 ; n, m+2 ; 2,-1\rangle+2 m|l,-m ; n, m+2 ; 2,-2\rangle \\
q_{3}(l, m, n)= & -c_{l, m, 2}|l,-m+1 ; n, m-2 ; 2,1\rangle+2 m|l,-m ; n, m-2 ; 2,2\rangle
\end{aligned}
$$

where,

$$
\begin{aligned}
& c_{l, m, 1}=\sqrt{(l-m)(l+m+1)}, \\
& c_{l, m, 2}=\sqrt{(l+m)(l-m+1)},
\end{aligned}
$$

the symbol $|n, k ; j, p ; l, m\rangle$ denotes the integral of the product of three spherical harmonics:

$$
\int_{\|\mathbf{m}\|=1} Y_{n}^{k} Y_{j}^{p} Y_{l}^{m} d \mathbf{m}
$$

(e.g., [25]). By the parity property of the spherical harmonics, any $a_{l, m}$ with odd $l$ is zero, and we only need to retain coefficients $a_{l, m}$ with nonnegative $m$. In simulations that generate Figures in the body, we assume constant $D_{r}$ to make contact with [7, 11]; all symmetry properties are valid for variable or constant rotary diffusivity.

\section{Reflection symmetry in simple shear.}

The dynamical system (47) provides an alternative form of the discrete reflection symmetry of $f$ in simple shear. To prove that these ODEs are invariant under the transformation

$$
\begin{aligned}
& a_{l, m} \longrightarrow-a_{l, m}, \quad l=2,4, \cdots, L_{\max }, \quad m \text { odd, } \\
& a_{l, m} \longrightarrow a_{l, m}, \quad l=2,4, \cdots, L_{\text {max }}, \quad m \text { even, }
\end{aligned}
$$

we split the variables $\left\{a_{l, m}\right\}$ 's into two groups, one having odd second index $m$, another even second index $m$. The first group of variables forms a vector $\mathbf{a}_{\mathbf{1}}$, and the second group of 
variables forms a vector $\mathbf{a}_{2}$. Then, the dynamical system (47) for the spherical harmonic amplitudes can be represented in the form

$$
\begin{aligned}
& \frac{d \mathbf{a}_{\mathbf{1}}}{d t}=\mathbf{F}_{\mathbf{1}}\left(\mathbf{a}_{\mathbf{1}}, \mathbf{a}_{2}\right) \\
& \frac{d \mathbf{a}_{\mathbf{2}}}{d t}=\mathbf{F}_{\mathbf{2}}\left(\mathbf{a}_{1}, \mathbf{a}_{2}\right) .
\end{aligned}
$$

The mirror symmetry follows if we can show that $\mathbf{F}_{\mathbf{1}}$ is an odd function of $\mathbf{a}_{\mathbf{1}}$ and $\mathbf{F}_{\mathbf{2}}$ is an even function of $\mathbf{a}_{\mathbf{1}}$. Since $\mathbf{F}_{\mathbf{1}}$ contains those functions $F_{l, m}^{j}$ with $m$ odd and $\mathbf{F}_{\mathbf{2}}$ contains those functions $F_{l, m}^{j}$ with $m$ even, we only need to check that $F_{l, m}^{j}$ is an odd function of $\mathbf{a}_{\mathbf{1}}$ for odd $m$ and $F_{l, m}^{j}$ is an even function of $\mathbf{a}_{\mathbf{1}}$ for even $m$. This is obviously true for the term $F_{l, m}^{1}$, because of the fact that $D_{r}(\mathbf{m}, a)$ is an even function of any variable $a_{l, m}$. For the second term, $F_{l, m}^{2}$ contains the product of the form

$$
a_{n, m-p} a_{2, p}
$$

For odd $m$, one and only one of $m-p$ and $p$ is odd. Therefore the product is an odd function of the first group of variables $\mathbf{a}_{\mathbf{1}}$. For even integer $m$, the integers $m-p$ and $p$ must be both even or both odd. So the product is an even function of the first group of variables $\mathbf{a}_{\mathbf{1}}$. The third term $F_{l, m}^{3}$ only contains linear terms. If $m$ is odd, then all of $a_{n, m}, a_{n, m+2}, a_{n, m-2}$ have odd second index, so $F_{l, m}^{3}$ is an odd function of the first group of variables $\mathbf{a}_{\mathbf{1}}$. On the other hand, if $m$ is even, then all of $a_{n, m}, a_{n, m+2}, a_{n, m-2}$ have even second index, so $F_{l, m}^{3}$ does not have any term relating to the first group of variables $\mathbf{a}_{\mathbf{1}}$. Therefore, this third term is an even function of $\mathbf{a}_{\mathbf{1}}$. These arguments establish the symmetry of solutions about the in-plane subspace formed by the first group of variables $\mathbf{a}_{\mathbf{1}}$, with the second group vanishing. 